\title{
Serious Leisure Perspectives in Sports: Professional Athletes' Career Progress via
} Serious Leisure

\author{
Ali Selman Özdemir \\ Department of Recreation, School of Physical Education and Sport, Cyprus International University, Nicosia, \\ Cyprus. \\ Email:ozdemiraliselman@gmail.com Tel:+905324845248
}

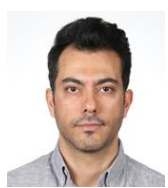

\begin{abstract}
The aim of this study is to determine serious leisure perspectives of professional athletes. 689 athletes selected through random sampling participated in the study. The data were collected with the "Serious Leisure Inventory and Measure (Short Form) (SLIM)", developed by Gould et al. (2011) adapted to Turkish by Ozdemir, Ayyıldız Durhan, and Akgül (2020) which consisting of 12 items and 3 sub-dimensions. It was determined that the data were not distributed homogeneously and non parametric tests were applied. In the analysis of the data, descriptive statistics, Man Whitney U, Kruskall Wallis, Tukey (HSD-LSD) and Pearson Correlation test were used for within-group comparisons. In this study, total internal reliability coefficient of SLIM (short form) scale was determined as .93. Participants had higher SLIM scores $(89,60 \pm 15,76)$, the highest subscale score was "identification with pursuit and social outcomes" sub-dimension (37,39 $\pm 7,35)$, and the lowest subscale score was "individual outcomes" (22,30 $\pm 4,39)$. Significant relationships and differences were found among the variables of gender, sports, age of athletics, number of trainings per week, participation status of international competitions, perceived challenge of duties, perceived skill development and perceived total development. As a result of the research, it was determined that professional athletes had high serious leisure perspectives and this situation being in interaction with certain variables.
\end{abstract}

Keywords: Serious leisure, Professional athletes, Career progress, Volleyball, Judo, Taekwondo.

Citation | Ali Selman Özdemir (2020). Serious Leisure Perspectives in Sports: Professional Athletes' Career Progress via Serious Leisure. Asian Journal of Education and Training, 6(2): 186-195. History:

Received: 20 December 2019

Revised: 3 February 2020

Accepted: 9 March 2020

Published: 80 March 2000

Licensed: This work is licensed under a Creative Commons

Attribution 3.0 License (oc)

Publisher: Asian Online Journal Publishing Group
Funding: This study received no specific financial support.

Competing Interests: The author declares that there are no conflicts of interests regarding the publication of this paper.

Transparency: The author confirms that the manuscript is an honest, accurate, and transparent account of the study was reported; that no vital features of the study have been omitted; and that any discrepancies from the study as planned have been explained.

Ethical: This study follows all ethical practices during writing.

\section{Contents}

1. Introduction

References... 


\section{Contribution of this paper to the literature}

This study contributes in the existing literature as a special example of 'career achieved through serious leisure pursuits' in terms of individuals starting their potential sports life at a young age primarily as a hobby, continuing as an amateur pursuit and reaching a professional career.

\section{Introduction}

The serious leisure perspective (SLP) is a typology of leisure activity developed and promulgated by Robert Stebbins as an ongoing project since 1974 (Stebbins, 2007; Veal, 2017). In the SLP, leisure is defined as uncoerced activity engaged in during free time, which people want to do and, in either a satisfying or a fulfilling way (or both), use their abilities and resources to succeed at this (Stebbins, 2007). The SLP is the theoretic framework that synthesizes three main forms of leisure, known as serious leisure (serious pursuits), casual leisure, and project-based leisure. Many of the roots of the SLP date to late 1973, even though the concept itself was only formally introduced and elaborated much later in Stebbins (Stebbins, 2015). Stebbins (2007) defined the distinctive features, similarities, and interrelationships of three forms of leisure. In his view, casual leisure is immediately, intrinsically rewarding, relatively short-lived pleasurable activity, requiring little or no special training to enjoy it. Project-based leisure is short-term, reasonably complicated, one-shot or occasional, though infrequent, creative undertaking carried out in free time, or time free of disagreeable obligation. Serious leisure is the systematic pursuit of an amateur, hobbyist, or volunteer core activity that people find so substantial, interesting, and fulfilling that, in a typical case, they launch themselves on a (leisure) career centered on acquiring and expressing a combination of its special skills, knowledge, and experience. Serious leisure activities require significant personal effort and even an occasional need to persevere. Serious leisure activities have durable, lasting benefits. Participants identify strongly with the activity and can even find a career in it, meaning that they experience different stages of achievement/involvement in the activity during their lifetime (Stebbins, 1996; Stebbins, 2009).

Six distinguishing qualities differentiate serious leisure from less substantial, short-term leisure pursuits (Stebbins, 2007; Stebbins, 1992; Stebbins, 2014). Identified six characteristics of serious leisure including: (1) persevere, (2) leisure career, (3) significant personal effort, (4) durable benefits, (5) unique ethos, and (6) strong identification with an activity (Stebbins, 1982). Participants embark upon a career in their activity and may need to occasionally persevere to enable ongoing participation. Significant personal effort is invested in the activity, developing knowledge, skills, and experience. Stebbins also maintains that ongoing participation in serious leisure is driven by the realization of durable benefits including self-actualization, self-enrichment, and/or self-gratification. Finally, participants engage with the unique ethos and social world of their activity and strongly identify with the activity in their projection of self to the world (Lamont, Kennelly, \& Moyle, 2014; Lamont, Kennelly, \& Moyle, 2019; Stebbins, 2014).

Stebbins (2001) regarded that serious leisure is the best way for people spending their free time in postmodern society. Within this type of leisure, people feel deep satisfaction and experience a full existence. In serious leisure, individuals' feels substantial and interesting in their leisure pursuits, acquire special skills, knowledge and experience through their leisure dedication and have a career development.

Career development in serious leisure is considered to be long-term development with special turning points or stages of involvement and personal commitment (Kraus, 2014; Raisborough, 2007; Stebbins, 1992). A career is 'the typical course, or passage, of certain types of amateur-professional practitioners that carries them into, and through, a leisure role ...' (Stebbins, 1992). Persons engaged in serious leisure, develop careers centered on their chosen activity (Stebbins, 2007) Serious leisure careers involve an individual's "steady development as a skilled, experienced, and knowledgeable participant” in a particular activity (Stebbins, 2007). Careers typically include five stages: beginning, development, establishment, maintenance and decline of special skills and knowledge (Stebbins, 2012). For an instance, in the case of adult amateur ice skaters, McQuarrie and Jackson (1996) identified five progressive leisure career stages: (1) becoming a potential participant; (2) beginning in the sport; (3) development; (4) establishment; and (5) decline or exit from the sport. The various characteristics associated with each of these leisure career stages are discussed in greater detail by Stebbins (2007). The beginning stage lasts as long as it is necessary for the activity to take root. Development occurs when the activity becomes more or less routine and systematic. A change to the establishment stage occurs when the participant has moved beyond learning the basics of the activity. During the maintenance stage, the leisure career is in "full bloom" and participants enjoy their pursuit to its utmost. In the final stage, not all participants reach the decline or exit stage, but in the case of those who do so, it may be due to deteriorating mental or physical skills. Some may then search for a new activity (Stebbins, 2007). Besides, Gould, Moore, McGuire, and Stebbins (2008) subdivided the career quality of serious leisure into two dimensions: career progress and career contingencies. Career progress focuses on the conscious improvement of leisure participation during the individuals' engagement. Career contingencies emphasize the turning points or special events of that leisure involvement.

Pursuing a career in sports as an athlete in a semi-professional or professional category, begins as a hobbyist or an amateur interest under the umbrella of serious leisure perspective. Achieving a career in sports through serious leisure; supported by school and collegiate sports as much as amateur sport clubs. However, weekend sports classes for early ages also play an important role to get a good start. Sport is also often a form of serious leisure (Stebbins.... 1992) that provides participants "small worlds" of social identification, opportunities for learning and developing skills, escape from monotonous and routine work, recognition for commitment and excellence, and a context for the expression of achievement motivations. Within the framework of serious leisure, a wide and diverse range of studies have been conducted over the past three decades with sports being one of the most popular activities in the domain of serious leisure, including master swimmers (Hastings, Kurth, Schloder, \& Cyr, 1995) youth sports (Siegenthaler \& Gonzalez, 1997) whitewater kayakers (Sherry \& Bartram, 2001) dancers (Brown, 2007) cycling (O'Connor \& Brown, 2010) playing chess (Gould et al., 2011) taekwondo participation (Kim, Dattilo, \& Heo, 2011) amateur athletes (MacCosham, Patry, Beswick, \& Gravelle, 2015) competition climbing (Gagnon, Stone, \& Garst, 2015) runners (Ronkainen, Harrison, Shuman, \& Ryba, 2017) female roller derby athletes (Liu, Baghurst, \& Bradley, 2018) and different sets of athletes (Romero, Iraurgi, Madariaga, \& Gould, 2020). These 
studies demonstrated a strong relationship between the serious leisure characteristics of athletes and their leisure involvement and dedication. Therefore, the purpose of the study is to examine the serious leisure perspectives of athletes in Turkey. Second it's aimed to understand relationship between serious leisure characteristics and career progress, and other variables.

\section{Methodology}

\subsection{Study Sample}

The sample of the study consists of 689 semi-professional and professional athletes whose playing volleyball, doing judo and taekwondo from several sports clubs in Turkey. The data were collected between March and June 2019 at the province of Ankara, İstanbul, Bursa and İzmir during the intercollegiate judo and taekwondo competitions, volleyball super leagues' play-off and play-out competitions in Turkey. In the collected data, 34 surveys were not evaluated in the research because of the missing. Aims of the study, the consent form, voluntary participation in the study, and confidentiality of the survey responses were verbally explained by investigators before the distribution of the surveys to subjects. The survey were answered and completed within an average of 15 minutes. Demographic information about the research group is given in Table 1.

\subsection{Instrumentation}

The data were collected with the "Serious Leisure Inventory and Measure (Short Form)" (SLIM), developed by Gould et al. (2011). SLIM adapted to Turkish testing of validity and reliability by Ozdemir et al. (2020) which consisting of 12 items and 3 sub-dimensions. The short form of Turkish version-SLIM includes three subdimensions as; "identification with pursuit and social outcomes", "perseverance and leisure career" and "individual outcomes." In addition, there are 8 more questions regarding the demographic variables and sports background of the athletes.

\subsection{Data Analysis}

It was determined that the data were not distributed homogeneously and non-parametric tests were applied. In the analysis of the data, descriptive statistics, Man Whitney U, Kruskall Wallis, Tukey (HSD-LSD) and Pearson Correlation tests were used for within-group comparisons. In this study, the total internal reliability coefficient of the SLIM (short form) scale was determined as .93. Data were analyzed by using SPSS 23.0 version.

\section{Results}

Table-1. Frequency and percentage range of demographic variables.

\begin{tabular}{|c|c|c|c|}
\hline \multicolumn{3}{|l|}{$\mathrm{n}=(689)$} & \multirow[b]{2}{*}{$\%$} \\
\hline & Variable & f & \\
\hline \multirow[t]{2}{*}{ Gender } & Female & 449 & 65,2 \\
\hline & Male & 240 & 34,8 \\
\hline \multirow[t]{3}{*}{ Sports } & Judo & 160 & 23,2 \\
\hline & Taekwondo & 342 & 49,6 \\
\hline & Volleyball & 187 & 27,1 \\
\hline \multirow[t]{10}{*}{ Age of Athletics } & 2 or 3 years or less & 32 & 4,6 \\
\hline & 4 or 5 years & 44 & 6,4 \\
\hline & 6 or 7 years & 67 & 9,7 \\
\hline & 8 or 9 years & 66 & 9,6 \\
\hline & 10 or 11 years & 92 & 13,4 \\
\hline & 12 or 13 years & 123 & 17,9 \\
\hline & 14 or 15 years & 89 & 12,9 \\
\hline & 16 or 17 years & 52 & 7,5 \\
\hline & 18 or 19 years & 82 & 11,9 \\
\hline & 20 years and above & 42 & 6,1 \\
\hline \multirow[t]{6}{*}{ Training per week } & 2 times per week or less & 105 & 15,2 \\
\hline & 3 times & 138 & 20,0 \\
\hline & 4 times & 71 & 10,3 \\
\hline & 5 times & 98 & 14,2 \\
\hline & 6 times & 113 & 16,4 \\
\hline & 7 times and above & 164 & 23,8 \\
\hline \multirow[t]{2}{*}{ Participation of International Competitions } & Participated & 401 & 58,2 \\
\hline & Non participated & 288 & 41,8 \\
\hline \multirow{8}{*}{ Perceived challenge of the duties (self scoring range of 1 to 10 ) } & 3 and less (point) & 59 & 8,6 \\
\hline & 4 & 66 & 9,6 \\
\hline & 5 & 115 & 16,7 \\
\hline & 6 & 94 & 13,6 \\
\hline & 7 & 126 & 18,3 \\
\hline & 8 & 118 & 17,1 \\
\hline & 9 & 49 & 7,1 \\
\hline & 10 & 62 & 9,0 \\
\hline \multirow[t]{6}{*}{ Perceived skill development (self scoring range of 1 to 10 ) } & 3 and less (point) & 42 & 6,1 \\
\hline & 4 & 43 & 6,2 \\
\hline & 5 & 97 & 14,1 \\
\hline & 6 & 94 & 13,6 \\
\hline & 7 & 156 & 22,6 \\
\hline & 8 & 144 & 20,9 \\
\hline
\end{tabular}




\begin{tabular}{|c|c|c|c|}
\hline & 9 & 52 & 7,5 \\
\hline & 10 & 61 & 8,9 \\
\hline \multirow[t]{3}{*}{ Perceived total development (self scoring range of 1 to 10 ) } & Better & 316 & 45,9 \\
\hline & Same & 256 & 37,2 \\
\hline & Worse & 117 & 17,0 \\
\hline
\end{tabular}

Table 1 provides information about athletes' demographic and professional profiles. Accordingly, the majority of the participants are women $(65.2 \%)$ and active athletes $(17.9 \%)$ for an average of $12-13$ years. In addition; the participants are taekwondo (49.6\%), volleyball $(27.1 \%)$ and judo $(23.2 \%)$ athletes respectively, who trains 7 or more times per week $(23.8 \%)$, participated in international competitions $(58.2 \%)$, consists of athletes who grades seven points both of perceived challenge degree (18.3\%) and skill development (2.6\%) out of ten, and express their perceived total development as "better" (45.9\%).

Table-2. Mean and standard deviation values of the SLIM.

\begin{tabular}{l|c|c|c|c}
\hline \multicolumn{2}{c|}{$\mathbf{n = ( 6 8 9 )}$} & & \\
\hline & Min. & Max. & $\bar{x}$ & ss \\
\hline SLIM & 16,00 & 108,00 & 89,60 & 15,76 \\
\hline Identification with pursuit and Social outcomes & 5,00 & 45,00 & 37,39 & 7,35 \\
\hline Perseverance and Leisure career & 4,00 & 36,00 & 29,89 & 5,64 \\
\hline Individual outcomes & 3,00 & 27,00 & 22,30 & 4,39 \\
\hline
\end{tabular}

As a result of the analyzes in Table 2 , it was determined that athletes have higher SLIM scores $(89,60 \pm 15,76)$, the highest subscale score was "identification with pursuit and social outcomes" sub-dimension (37,39 $\pm 7,35)$, and the lowest subscale score was "individual outcomes" (22,30 \pm 4,39).

Table-3. Man Whitney U test results between participants' gender and SLIM.

\begin{tabular}{l|c|c|c|c|c|c}
\hline & Gender & $\mathbf{n}$ & Mean rank & Rank sum & $\mathbf{z}$ & $\mathbf{p}$ \\
\hline \multirow{2}{*}{ SLIM } & Female & 449 & 355,70 & 159710,00 & $-1,931$ & 0,053 \\
\cline { 2 - 7 } & Male & 240 & 324,98 & 77995,00 & & \\
\hline \multirow{2}{*}{$\begin{array}{l}\text { Identification with pursuit and Social } \\
\text { outcomes }\end{array}$} & Female & 449 & 354,85 & 159326,50 & $-1,782$ & 0,075 \\
\cline { 2 - 7 } & Male & 240 & 326,58 & 78378,50 & & \\
\hline \multirow{2}{*}{$\begin{array}{l}\text { Perseverance and Leisure career } \\
\text { Individual outcomes }\end{array}$} & Female & 449 & 353,78 & 158845,00 & $-1,589$ & 0,112 \\
\cline { 2 - 7 } & Male & 240 & 328,58 & 78860,00 & & $0,038^{*}$ \\
\hline
\end{tabular}

Note: $* \mathrm{p}<0,05$

When the Man Whitney U test results were examined between the gender variable and SLIM in Table 3, it was seen that there is a significant difference between the Individual outcomes sub-dimensions and the gender variable. In the Individual outcomes sub-dimension, it was determined that women athletes displayed higher development scores than men. There was no significant relationship between total scores and other sub-dimensions and gender.

Table-4. Kruskall Wallis test results between sports and SLIM.

\begin{tabular}{|c|c|c|c|c|c|c|}
\hline & Sports & $\mathbf{n}$ & Mean rank & df & $\mathrm{x}^{2}$ & p \\
\hline \multirow[t]{4}{*}{ SLIM } & $\mathrm{Judo}^{\mathrm{c}}$ & 160 & 323,68 & 2 & \multirow[t]{5}{*}{12,294} & \multirow[t]{5}{*}{$0,002 *$} \\
\hline & Taekwondo & 342 & 331,32 & & & \\
\hline & Volleyballa $^{\mathrm{a}}$ & 187 & 388,25 & & & \\
\hline & Total & 689 & & & & \\
\hline \multirow{4}{*}{$\begin{array}{l}\text { Identification with pursuit } \\
\text { and Social outcomes }\end{array}$} & Judo & 160 & 325,22 & 2 & & \\
\hline & Taekwondo & 342 & 338,79 & & \multirow[t]{3}{*}{5,731} & \multirow[t]{3}{*}{0,057} \\
\hline & Volleyball & 187 & 373,29 & & & \\
\hline & Total & 689 & & & & \\
\hline \multirow{4}{*}{$\begin{array}{l}\text { Perseverance and Leisure } \\
\text { career }\end{array}$} & Judo $^{b}$ & 160 & 326,32 & 2 & & \\
\hline & Taekwondo ${ }^{c}$ & 342 & 325,63 & & 17,261 & O,000* \\
\hline & Volleyballa $^{\mathrm{a}}$ & 187 & 396,40 & & & \\
\hline & Total & 689 & & & & \\
\hline \multirow[t]{4}{*}{ Individual outcomes } & Judo $^{\mathrm{b}}$ & 160 & 338,07 & 2 & & \\
\hline & Taekwondo $^{c}$ & 342 & 330,27 & & & \\
\hline & Volleyballa $^{\mathrm{a}}$ & 187 & 377,86 & & 7,253 & $0,027 *$ \\
\hline & Total & 689 & & & & \\
\hline
\end{tabular}

According to the Kruskall Wallis test results between sports variable and SLIM in Table 4, it was observed that significant differences between in sub-dimensions and total scores of SLIM. Accordingly, it has been determined that professional athletes in the volleyball branch gave higher SLIM levels compared to the participants in other branches. 
Table-5. Kruskall Wallis test results between age of athletics and SLIM.

\begin{tabular}{|c|c|c|c|c|c|c|}
\hline & Age of athletics & $\mathbf{n}$ & Mean rank & df & $x^{2}$ & $\mathbf{p}$ \\
\hline \multirow[t]{11}{*}{ SLIM } & 2 or 3 years or less ${ }^{c}$ & 32 & 254,94 & 9 & \multirow[t]{11}{*}{24,163} & \multirow[t]{11}{*}{$0,004 *$} \\
\hline & 4 or 5 years & 44 & 286,19 & & & \\
\hline & 6 or 7 years & 67 & 305,21 & & & \\
\hline & 8 or 9 years & 66 & 320,94 & & & \\
\hline & 10 or 11 years & 92 & 325,65 & & & \\
\hline & 12 or 13 years & 123 & 371,57 & & & \\
\hline & 14 or 15 years $^{b}$ & 89 & 370,70 & & & \\
\hline & 16 or 17 years $^{\mathrm{a}}$ & 52 & 393,90 & & & \\
\hline & 18 or 19 years & 82 & 369,44 & & & \\
\hline & 20 years and above & 42 & 378,37 & & & \\
\hline & Total & 689 & & & & \\
\hline \multirow{11}{*}{$\begin{array}{l}\text { Identification with pursuit } \\
\text { and Social outcomes }\end{array}$} & 2 or 3 years or less & 32 & 254,02 & 9 & \multirow[t]{3}{*}{17,257} & \multirow[t]{3}{*}{$0,045^{*}$} \\
\hline & 4 or 5 years & 44 & 298,38 & & & \\
\hline & 6 or 7 years & 67 & 320,07 & & & \\
\hline & 8 or 9 years & 66 & 325,00 & & & \\
\hline & 10 or 11 years & 92 & 341,33 & & & \\
\hline & 12 or 13 years $^{\mathrm{c}}$ & 123 & 361,75 & & & \\
\hline & 14 or 15 years $^{d}$ & 89 & 351,52 & & & \\
\hline & 16 or 17 years $^{\mathrm{a}}$ & 52 & 396,10 & & & \\
\hline & 18 or 19 years $^{b}$ & 82 & 372,66 & & & \\
\hline & 20 years and above & 42 & 362,27 & & & \\
\hline & Total & 689 & & & & \\
\hline \multirow{11}{*}{$\begin{array}{l}\text { Perseverance and Leisure } \\
\text { career }\end{array}$} & 2 or 3 years or less & 32 & 262,83 & 9 & 23,299 & $0,006^{*}$ \\
\hline & 4 or 5 years & 44 & 285,38 & & & \\
\hline & 6 or 7 years & 67 & 306,81 & & & \\
\hline & 8 or 9 years & 66 & 332,36 & & & \\
\hline & 10 or 11 years & 92 & 314,28 & & & \\
\hline & 12 or 13 years $^{\mathrm{a}}$ & 123 & 375,92 & & & \\
\hline & 14 or 15 years $^{\mathrm{c}}$ & 89 & 372,60 & & & \\
\hline & 16 or 17 years $^{b}$ & 52 & 375,44 & & & \\
\hline & 18 or 19 years & 82 & 368,51 & & & \\
\hline & 20 years and above & 42 & 385,51 & & & \\
\hline & Total & 689 & & & & \\
\hline \multirow[t]{11}{*}{ Individual outcomes } & 2 or 3 years or less ${ }^{b}$ & 32 & 259,28 & 9 & 23,756 & $0,000^{*}$ \\
\hline & 4 or 5 years & 44 & 298,75 & & & \\
\hline & 6 or 7 years & 67 & 305,37 & & & \\
\hline & 8 or 9 years & 66 & 307,67 & & & \\
\hline & 10 or 11 years & 92 & 328,32 & & & \\
\hline & 12 or 13 years & 123 & 365,85 & & & \\
\hline & 14 or 15 years $^{a}$ & 89 & 388,70 & & & \\
\hline & 16 or 17 years & 52 & 386,64 & & & \\
\hline & 18 or 19 years & 82 & 368,14 & & & \\
\hline & 20 years and above & 42 & 366,80 & & & \\
\hline & Total & 689 & & & & \\
\hline
\end{tabular}

When the Kruskall Wallis test results examined between the age of athletics and SLIM, it was seen significant differences between in sub-dimensions and total scores of SLIM. Accordingly, it has been determined that the groups with 12 and more age of athletics years have higher averages than the other groups in the significant difference observed in SLIM total scores and sub-dimension scores. Differences in the within-group were tested by Tukey HSD and LSD tests and shown in Table 5.

Table-6. Kruskall Wallis test results between number of training per week and SLIM

\begin{tabular}{|c|c|c|c|c|c|c|}
\hline & Number of training per week & $\mathbf{n}$ & Mean rank & df & $\mathrm{x}^{2}$ & $\mathbf{p}$ \\
\hline \multirow[t]{7}{*}{ SLIM } & 2 times or less ${ }^{\mathrm{f}}$ & 105 & 266,21 & 5 & \multirow[t]{7}{*}{54,452} & \multirow[t]{7}{*}{$0,000^{*}$} \\
\hline & 3 times $^{\mathrm{e}}$ & 138 & 275,73 & & & \\
\hline & 4 times $^{b}$ & 71 & 382,98 & & & \\
\hline & 5 times $^{\mathrm{d}}$ & 98 & 368,19 & & & \\
\hline & 6 times $^{\mathrm{c}}$ & 113 & 369,93 & & & \\
\hline & 7 times and above ${ }^{a}$ & 164 & 406,26 & & & \\
\hline & Total & 689 & & & & \\
\hline \multirow{7}{*}{$\begin{array}{l}\text { Identification with pursuit } \\
\text { and Social outcomes }\end{array}$} & 2 times or less ${ }^{c}$ & 105 & 296,95 & 5 & \multirow[t]{3}{*}{18,968} & \multirow[t]{3}{*}{$0,002^{*}$} \\
\hline & 3 times $^{\mathrm{b}}$ & 138 & 307,42 & & & \\
\hline & 4 times & 71 & 366,08 & & & \\
\hline & 5 times & 98 & 355,36 & & & \\
\hline & 6 times & 113 & 356,33 & & & \\
\hline & 7 times and above ${ }^{\mathrm{a}}$ & 164 & 384,25 & & & \\
\hline & Total & 689 & & & & \\
\hline \multirow{4}{*}{$\begin{array}{l}\text { Perseverance and Leisure } \\
\text { career }\end{array}$} & 2 times or less ${ }^{\mathrm{f}}$ & 105 & 238,70 & 5 & 92,558 & $0,000^{*}$ \\
\hline & 3 times $^{\mathrm{e}}$ & 138 & 253,86 & & & \\
\hline & 4 times $^{\mathrm{c}}$ & 71 & 396,11 & & & \\
\hline & 5 times $^{\mathrm{d}}$ & 98 & 386,76 & & & \\
\hline
\end{tabular}




\begin{tabular}{|c|c|c|c|c|c|c|}
\hline & 6 times $^{\mathrm{b}}$ & 113 & 392,37 & & & \\
\hline & 7 times and above ${ }^{\mathrm{a}}$ & 164 & 410,03 & & & \\
\hline & Total & 689 & & & & \\
\hline \multirow[t]{7}{*}{ Individual outcomes } & 2 times or less ${ }^{\mathrm{c}}$ & 105 & 284,17 & 5 & 36,277 & $0,000^{*}$ \\
\hline & 3 times $^{\mathrm{b}}$ & 138 & 288,78 & & & \\
\hline & 4 times & 71 & 367,41 & & & \\
\hline & 5 times & 98 & 359,71 & & & \\
\hline & 6 times & 113 & 363,15 & & & \\
\hline & 7 times and above & 164 & 400,26 & & & \\
\hline & Total & 689 & & & & \\
\hline
\end{tabular}

According to Kruskall Wallis test results between weekly training number and measurement tools in Table 6, it was determined that there were significant relationships between SLIM and variables. Accordingly, while the number of weekly trainings increases, SLIM level increases.

\begin{tabular}{|c|c|c|c|c|c|c|}
\hline & $\begin{array}{l}\text { Participation of } \\
\text { International } \\
\text { Competitions }\end{array}$ & $\mathbf{n}$ & Mean rank & $\begin{array}{c}\text { Rank } \\
\text { sum }\end{array}$ & $\bar{z}$ & $\mathbf{p}$ \\
\hline \multirow[t]{2}{*}{$\overline{\text { SLIM }}$} & Participant & 401 & 376,89 & 151133,00 & $-4,965$ & $0,000^{*}$ \\
\hline & Nonparticipant & 288 & 300,60 & 86572,00 & & \\
\hline \multirow{2}{*}{$\begin{array}{l}\text { Identification with pursuit } \\
\text { and Social outcomes }\end{array}$} & Participant & 401 & 359,73 & 144253,50 & $-2,300$ & $0,021 *$ \\
\hline & Nonparticipant & 288 & 324,48 & 93451,50 & & \\
\hline \multirow{2}{*}{$\begin{array}{l}\text { Perseverance and Leisure } \\
\text { career }\end{array}$} & Participant & 401 & 390,65 & 156652,50 & $-7,134$ & O,OOO* \\
\hline & Nonparticipant & 288 & 281,43 & 81052,50 & & \\
\hline \multirow[t]{2}{*}{ Individual outcomes } & Participant & 401 & 375,66 & 150638,00 & $-4,800$ & O,OOO* \\
\hline & Nonparticipant & 288 & 302,32 & 87067,00 & & \\
\hline
\end{tabular}

Table-8. Kruskall Wallis test results between challenge of the duties and SLIM.

\begin{tabular}{|c|c|c|c|c|c|c|}
\hline & $\begin{array}{c}\text { Perceived challenge of the } \\
\text { duties (point) }\end{array}$ & $\mathbf{n}$ & Mean rank & df & $\mathrm{x}^{2}$ & $\mathbf{p}$ \\
\hline \multirow{9}{*}{$\begin{array}{l}\text { SLIM (self scoring range of } 1 \text { to } \\
\text { 10) }\end{array}$} & 3 or less & 59 & 377,21 & 7 & \multirow[t]{9}{*}{22,334} & \multirow[t]{9}{*}{$0,002 *$} \\
\hline & $4^{\mathrm{c}}$ & 66 & 343,76 & & & \\
\hline & $5^{\mathrm{e}}$ & 115 & 329,76 & & & \\
\hline & $6^{\mathrm{d}}$ & 94 & 337,69 & & & \\
\hline & $7 \mathrm{f}$ & 126 & 322,69 & & & \\
\hline & $8 \mathrm{~g}$ & 118 & 309,35 & & & \\
\hline & $9^{\text {a }}$ & 49 & 438,21 & & & \\
\hline & $10^{b}$ & 62 & 394,56 & & & \\
\hline & Total & 689 & & & & \\
\hline \multirow{9}{*}{$\begin{array}{l}\text { Identification with pursuit and } \\
\text { Social outcomes }\end{array}$} & 3 or less $^{b}$ & 59 & 381,01 & 7 & \multirow[t]{3}{*}{16,933} & \multirow[t]{3}{*}{$0,018^{*}$} \\
\hline & $4^{\mathrm{h}}$ & 66 & 316,73 & & & \\
\hline & $5^{\mathrm{f}}$ & 115 & 330,94 & & & \\
\hline & $6^{g}$ & 94 & 330,64 & & & \\
\hline & $7^{\mathrm{e}}$ & 126 & 341,50 & & & \\
\hline & $8^{\mathrm{d}}$ & 118 & 321,88 & & & \\
\hline & $9^{\mathrm{a}}$ & 49 & 434,82 & & & \\
\hline & $10^{c}$ & 62 & 368,81 & & & \\
\hline & Total & 689 & & & & \\
\hline \multirow[t]{9}{*}{ Perseverance and Leisure career } & 3 or less ${ }^{b}$ & 59 & 398,42 & 7 & 31,411 & O,OOO* \\
\hline & $4^{\mathrm{c}}$ & 66 & 339,11 & & & \\
\hline & 5 & 115 & 341,40 & & & \\
\hline & 6 & 94 & 348,13 & & & \\
\hline & $7^{\mathrm{e}}$ & 126 & 293,00 & & & \\
\hline & $8^{d}$ & 118 & 310,28 & & & \\
\hline & $9^{a}$ & 49 & 414,87 & & & \\
\hline & 10 & 62 & 418,92 & & & \\
\hline & Total & 689 & & & & \\
\hline \multirow[t]{9}{*}{ Individual outcomes } & 3 or less & 59 & 352,90 & 7 & 23,555 & $0,001 *$ \\
\hline & 4 & 66 & 387,25 & & & \\
\hline & $5^{\mathrm{b}}$ & 115 & 323,43 & & & \\
\hline & 6 & 94 & 339,36 & & & \\
\hline & 7 & 126 & 326,76 & & & \\
\hline & 8 & 118 & 301,59 & & & \\
\hline & $9^{\mathrm{a}}$ & 49 & 422,92 & & & \\
\hline & 10 & 62 & 399,17 & & & \\
\hline & Total & 689 & & & & \\
\hline
\end{tabular}


When Table 7 is examined, a statistically significant relationship was found in favor of those who participated in international competitions in all sub-dimensions and total scores of SLIM. Therefore, participating in international competitions should be said to change the SLIM level positively compared to those who did not.

According to Kruskall Wallis test results between perceived challenge of the duties and measurement tools in Table 8, it was determined that there were significant relationships between SLIM and variables. Therefore, it was seen that the participants who described the self score of perceived challenge of the duties 9, showed a higher SLIM level compared to the other grades.

Table-9. Kruskall Wallis test results between perceived skill development and SLIM.

\begin{tabular}{|c|c|c|c|c|c|c|}
\hline & $\begin{array}{c}\text { Perceived skill } \\
\text { development (point) }\end{array}$ & $\bar{n}$ & Mean rank & df & $\mathrm{x}^{2}$ & $\mathbf{p}$ \\
\hline \multirow{9}{*}{$\begin{array}{l}\text { SLIM (self-scoring range } \\
\text { of } 1 \text { to } 10 \text { ) }\end{array}$} & 3 or less 9 & 42 & 290,64 & 7 & \multirow[t]{9}{*}{42,205} & \multirow[t]{9}{*}{$0,000^{*}$} \\
\hline & $4^{f}$ & 43 & 271,73 & & & \\
\hline & 5 & 97 & 304,90 & & & \\
\hline & $6^{\mathrm{d}}$ & 94 & 325,61 & & & \\
\hline & $7 \mathrm{e}$ & 156 & 320,63 & & & \\
\hline & $8^{\mathrm{c}}$ & 144 & 381,03 & & & \\
\hline & $9^{b}$ & 52 & 427,57 & & & \\
\hline & $10^{\text {a }}$ & 61 & 434,61 & & & \\
\hline & Total & 689 & & & & \\
\hline \multirow{9}{*}{$\begin{array}{l}\text { Identification with pursuit } \\
\text { and Social outcomes }\end{array}$} & 3 or less & 42 & 317,10 & 7 & \multirow[t]{3}{*}{25,453} & \multirow[t]{3}{*}{ O,OO 1* } \\
\hline & $4^{\mathrm{e}}$ & 43 & 281,98 & & & \\
\hline & 5 & 97 & 330,78 & & & \\
\hline & $6^{\mathrm{d}}$ & 94 & 325,79 & & & \\
\hline & 7 & 156 & 316,88 & & & \\
\hline & $8^{c}$ & 144 & 371,25 & & & \\
\hline & $9^{\mathrm{b}}$ & 52 & 412,12 & & & \\
\hline & $10^{\mathrm{a}}$ & 61 & 413,61 & & & \\
\hline & Total & 689 & & & & \\
\hline \multirow{9}{*}{$\begin{array}{l}\text { Perseverance and Leisure } \\
\text { career }\end{array}$} & 3 or less ${ }^{f}$ & 42 & 283,10 & 7 & 56,055 & $0,000^{*}$ \\
\hline & $4 \mathrm{~g}$ & 43 & 261,12 & & & \\
\hline & $5^{\mathrm{e}}$ & 97 & 287,84 & & & \\
\hline & $6^{\mathrm{d}}$ & 94 & 317,81 & & & \\
\hline & $7^{\mathrm{d}}$ & 156 & 329,49 & & & \\
\hline & $8^{c}$ & 144 & 384,41 & & & \\
\hline & $9^{\text {a }}$ & 52 & 453,07 & & & \\
\hline & $10^{b}$ & 61 & 434,06 & & & \\
\hline & Total & 689 & & & & \\
\hline \multirow[t]{9}{*}{ Individual outcomes } & 3 or less 8 & 42 & 281,26 & 7 & 31,592 & $0,000^{*}$ \\
\hline & $4^{\mathrm{f}}$ & 43 & 292,80 & & & \\
\hline & 5 & 97 & 316,31 & & & \\
\hline & $6^{d}$ & 94 & 351,30 & & & \\
\hline & $7 \mathrm{e}$ & 156 & 314,91 & & & \\
\hline & $8^{c}$ & 144 & 369,08 & & & \\
\hline & $9^{\mathrm{b}}$ & 52 & 402,76 & & & \\
\hline & $10^{\mathrm{a}}$ & 61 & 432,46 & & & \\
\hline & Total & 689 & & & & \\
\hline
\end{tabular}

Table-10. Kruskall Wallis test results between perceived total development and SLIM

\begin{tabular}{|c|c|c|c|c|c|c|}
\hline & Perceived total development & $\mathbf{n}$ & Mean rank & df & $\mathrm{x}^{2}$ & $\mathbf{p}$ \\
\hline \multirow[t]{4}{*}{$\overline{\text { SLIM }}$} & Better $^{\mathrm{a}}$ & 316 & 406,44 & 2 & \multirow[t]{5}{*}{56,100} & \multirow[t]{5}{*}{$0,000^{*}$} \\
\hline & Same $^{b}$ & 256 & 297,43 & & & \\
\hline & Worse $^{c}$ & 117 & 283,15 & & & \\
\hline & Total & 689 & & & & \\
\hline \multirow{4}{*}{$\begin{array}{l}\text { Identification with pursuit and } \\
\text { Social outcomes }\end{array}$} & Better ${ }^{a}$ & 316 & 389,83 & 2 & & \\
\hline & Same $^{\mathrm{c}}$ & 256 & 306,76 & & \multirow[t]{3}{*}{29,811} & \multirow[t]{3}{*}{$0,000^{*}$} \\
\hline & Worse $^{b}$ & 117 & 307,59 & & & \\
\hline & Total & 689 & & & & \\
\hline \multirow[t]{4}{*}{ Perseverance and Leisure career } & Better ${ }^{a}$ & 316 & 417,23 & 2 & & \\
\hline & Same $^{b}$ & 256 & 294,08 & & 79,695 & $0, \mathrm{OOO}^{*}$ \\
\hline & Worse $^{c}$ & 117 & 261,34 & & & \\
\hline & Total & 689 & & & & \\
\hline \multirow[t]{4}{*}{ Individual outcomes } & Better ${ }^{a}$ & 316 & 396,98 & 2 & & \\
\hline & Same $^{c}$ & 256 & 299,90 & & & \\
\hline & Worse $^{b}$ & 117 & 303,28 & & 40,336 & $0,000^{*}$ \\
\hline & Total & 689 & & & & \\
\hline
\end{tabular}

Note: ${ }^{\mathrm{p}}<0,05$.

When the Kruskall Wallis test results examined between the perceived skill development level and SLIM in Table 9, it was seen that significant differences between in sub-dimensions and total scores of SLIM. Accordingly, it was observed that the participants who stated their perceived skill development levels' as increased between 9 and 10, showed higher SLIM levels compared to the other grades. 
According to Kruskall Wallis test results between perceived total development level and measurement tools in Table 10, it was seen that there were significant relationships between SLIM and variables. Therefore, it was understood that the participants who described the perceived total development level as "better", showed a higher SLIM level compared to the other groups.

\section{Discussion}

The main purpose of this study is to clarify serious leisure perspectives of semi-professional and professional athletes. In addition, it's aimed to determine the relationship and differences between serious leisure characteristics with career progress (perceived skill development and total development) and some variables.

As a result of the analyzes, it was determined that athletes have higher serious leisure scores. The highest subscale score was "identification with pursuit and social outcomes" and the lowest subscale score was "individual outcomes". Research into serious leisure has illustrated that the chosen activity becomes a central aspect of the participants' lives: 'it is time, resource, and therefore identity intensive' (Gillespie, Leffler, \& Lerner, 2002). It has also been argued that identity should be considered the central concept for understanding serious leisure, whereas the other five characteristics could be outcomes of developing this identity (Jones, 2006; Shipway \& Jones, 2007). Findings of an another study suggest that serious leisure pursuers in sport are in a central life interest as they were fully invested physically and mentally to their activity (Dubin, 1992). Heo and King (2009) found that serious sport participants tended to invest personal efforts and perseverance through the training process and dedicated their time to travel for various tournaments outside of their community. In different studies found that unique ethos influences participants' practices in yoga (Patterson, Getz, \& Gubb, 2016) and marathon (Robinson, Patterson, \& Axelsen, 2014) accordingly unique ethos enables participants at various levels to perceive their experiences as autonomy-supportive.

It was seen that the gender differences between serious leisure scores, there is a significant difference between the Individual outcomes sub-dimensions and the gender variable. In the Individual outcomes sub-dimension, it was determined that women athletes displayed higher development scores than men. In the study of Dilley and Scraton (2010) on women climbers as serious leisure, they indicate that: The women climbers identified their climbing as a serious leisure pursuit clearly demonstrating many of the qualities Stebbins has characterized as defining serious leisure. They pursued their climbing systematically, demonstrated perseverance, acquired specialist skills and knowledge, identified individual benefits they had gained such as belonging and feelings of accomplishment and they certainly associated with their activity. However, the data suggest that serious leisure is about far more than the activity itself. Their leisure is centrally about creating, negotiating and/or resisting ideological expectations (about physicality, motherhood) and creating individual spaces to 'be' and social spaces to 'belong' and be 'different'.

According to results between sports' branch and serious leisure scores, it was observed that significant differences between in sub-dimensions and total scores of SLIM. Accordingly, it has been determined that professional athletes in the volleyball branch gave higher serious leisure levels compared to the participants in other branches. According to Cheng and Tsaur (2012) unique ethos, strong identity with the activity, significant personal efforts and career pursuits in their endeavors are strong indicators of surfers' serious leisure characteristics. Generally, surfers with higher serious leisure characteristics have a higher recreation involvement. In other words, serious leisure attributes are the antecedents of recreation involvement. In addition; findings of the relationships among serious leisure and recreation involvement dimensions show that strong identity with the activity, perseverance, career pursuits in their endeavors and unique ethos are significantly associated with attraction. This result indicates that surfers with serious leisure characteristics can strongly identify with the activity, demonstrate perseverance in the activity, and endeavors under adversity.

When the test results examined between the perceived skill development level and serious leisure scores, it was seen that significant differences between in sub-dimensions and total scores of SLIM. Accordingly, it was observed that the participants who stated their perceived skill development levels' as increased between 9 and 10, showed higher SLIM levels compared to the other grades. A study of participation in adaptive sports as a serious leisure pursuit indicated a positive relationship between participants' self-determination and skill level. Further, sport skill development was related to participants gaining social rewards and physical improvements, as well as enhancing their self-determination to pursue serious leisure (Jinmoo Heo, Lee, Lundberg, McCormick, \& Chun, 2008). In a different study, Lewis, Patterson, and Pegg (2013) states as; motorcycle road racing becomes a more serious leisure activity as racers become more committed to regular competition, increasing their skill levels and seeking membership in motorcycle clubs. For the young racer in particular, there is a growing commitment to develop higher level skills a means of self-improvement which can ultimately lead to a career pathway in this sport for those who seek it.

According to test results between perceived total development level and serious leisure level, it was understood that the participants who described the perceived total development level as "better", showed a higher SLIM level compared to the other groups. According to Ronkainen et al. (2017) commitment, high levels of effort, hard work and acceptance of pain and discomfort, are often central values for serious runners. In the study of Hungling, Caneday, and Tapps (2013) it was found that community sports program participants' strong identification of serious leisure was the most robust indicator reflecting the amateur athletes' seriousness of their leisure pursuit. In a different research, it's mentioned that serious leisure participants often discover leisure careers that reflect stages of experience, such as "occasional dancers," "wannabes," and "hard-core dancers" or "therapeutic players," "social golfers,” “moderate devotees” and “core devotees” (Heo, Lee, Kim, \& Stebbins, 2012; Siegenthaler \& O’Dell, 2003).

\section{Conclusion}

Sports, exercise and physical activities have an important place among serious leisure activities. In terms of being easily accessible, easy participation, perseverance, effort, competition, entertainment, self-expression and socialization; It is an area where individuals can establish strong unique ethos with the sport they are dealing with and feel belonging and devotion. 
The individual, who started the sport at an early age for hobby purposes, or guided by family for avoiding bad habits, by emulating of his peers or influenced by the team he support, or discovered in an organized talent selection, has experienced six qualities of Stebbins' for serious leisure. In line with these qualities, that can be experienced as a hobbyist, amateur or volunteer. Therefore it could be thought that; the individual who shows high effort and perseverance for his sports, who is devoted to it and who does not give up in moments of breaking with certain turning points, has achieved a career in sports via serious leisure, at some point of this journey.

This study measured the serious leisure levels of semi-professional and professional athletes who had reached a certain career through their own serious leisure pursues, and found positive relationships between all the subdimensions and parameters in the scale that represent the characteristics of the serious leisure perspective. In the light of the explanations above, based on the findings of this study, it can be said that pursuing a career in professional sports is associated with the serious leisure theory and that the career process in sports can be explained with the serious leisure perspective.

Due to their camps and intense competition schedule, the difficulty of reaching the athletes and having a meeting within the scope of the research was among the most important limitations of this study. In the future researches, different sports branches can be discussed and international comparisons can be made.

\section{References}

Brown, C. A. (2007). The carolina shaggers: Dance as serious leisure. Journal of Leisure Research, 39(4), 623-647.Available at: https://doi.org/10.1080/00222216.2007.11950125.

Cheng, T.-M., \& Tsaur, S.-H. (2012). The relationship between serious leisure characteristics and recreation involvement: A case study of Taiwan's surfing activities. Leisure Studies, 31(1), 53-68.Available at: https://doi.org/10.1080/02614367.2011.568066.

Dilley, R. E., \& Scraton, S. J. (2010). Women, climbing and serious leisure. Leisure Studies, 29(2), 125-141.

Dubin, R. (1992). Central life interests: Creative individualism in a complex world. New Brunswick, NJ: Transaction.

Gagnon, R. J., Stone, G. A., \& Garst, B. A. (2015). Competition climbing as serious leisure. Paper presented at the Paper presented at the Proceedings from the Northeastern Recreation Research Symposium 2015, Annapolis, MD.

Gillespie, D. L., Leffler, A., \& Lerner, E. (2002). If it weren 't for my hobby, I'd have a life: Dog sports, serious leisure, and boundary negotiations. Leisure Studies, 21(3-4), 285-304.Available at: https://doi.org/10.1080/0261436022000030632.

Gould, J., Moore, D., Karlin, N. J., Gaede, D. B., Walker, J., \& Dotterweich, A. R. (2011). Measuring serious leisure in chess: Model confirmation and method bias. Leisure Sciences, 33(4), 332-340.Available at: https://doi.org/10.1080/01490400.2011.583165.

Gould, J., Moore, D., McGuire, F., \& Stebbins, R. (2008). Development of the serious leisure inventory and measure. Journal of Leisure Research, 4O(1), 47-68.Available at: https://doi.org/10.1080/002222 16.2008.11950132.

Hastings, D. W., Kurth, S. B., Schloder, M., \& Cyr, D. (1995). Reasons for participating in a serious leisure career: Comparison of Canadian and US masters swimmers. International Review for the Sociology of Sport, 30(1), 101-119.Available at: https://doi.org/10.1177/101269029503000106.

Heo, J., \& King, C. (2009). Senior games as serious sport tourism. Journal of Research, 4(2), 6-9.

Heo, J., Lee, Y., Lundberg, N., McCormick, B., \& Chun, S. (2008). Adaptive sport as serious leisure: Do self-determination, skill level, and leisure constraints matter. Annual in Therapeutic Recreation, 16, 31-38.

Heo, J., Lee, I. H., Kim, J., \& Stebbins, R. A. (2012). Understanding the relationships among central characteristics of serious leisure: An empirical study of older adults in competitive sports. Journal of Leisure Research, 44(4), 450-462.Available at: https://doi.org/10.1080/00222216.2012.11950273.

Hungling, S., Caneday, L., \& Tapps, T. (2013). An exploratory study of serious leisure and lifestyle for amateur athletes. LARNet: The Cyber Journal of Applied Leisure and Recreation Research, $16(3), 13-22$.

Jones, I. (2006). Examining the characteristics of serious leisure from a social identity perspective. In S. Elkington, I. Jones, \& L. Lawrence (Eds.), Serious leisure: Extensions and Applications (pp. 47-60).

Kim, J., Dattilo, J., \& Heo, J. (2011). Taekwondo participation as serious leisure for life satisfaction and health. Journal of Leisure Research, 43(4), 545-559.Available at: https://doi.org/10.1080/00222216.2011.11950249.

Kraus, R. (2014). Becoming a belly dancer: Gender, the life course and the beginnings of a serious leisure career. Leisure Studies, 33(6), 565579.Available at: https://doi.org/10.1080/02614367.2013.833284.

Lamont, M., Kennelly, M., \& Moyle, B. (2014). Costs and perseverance in serious leisure careers. Leisure Sciences, 36(2), 144-160.Available at: https://doi.org/10.1080/01490400.2013.857623.

Lamont, M., Kennelly, M., \& Moyle, B. (2019). Perspectives of endurance athletes' Spouses: A paradox of serious leisure. Leisure Sciences, 41(6), 477-498.Available at: https://doi.org/10.1080/01490400.2017.1384943.

Lewis, J., Patterson, I., \& Pegg, S. (2013). The serious leisure career hierarchy of Australian motorcycle road racers. World Leisure Journal, 55(2), 179-192.Available at: https://doi.org/10.1080/04419057.2013.782563.

Liu, H.-L. S., Baghurst, T., \& Bradley, M. (2018). Female roller derby athletes' athletic identity and systematic pursuit of leisure. Journal of Amateur Sport, 4(1), 108-128.Available at: https://doi.org/10.17161/jas.v4i 1.6702.

MacCosham, B., Patry, P., Beswick, C., \& Gravelle, F. (2015). Leisure lifestyle and dropout: Exploring the experience of amateur athletes in competitive sport. International Journal of Sport Management, Recreation and Tourism, 20(2), 20-39.Available at: https://doi.org/10.5199/ijsmart-1791-874x-20b.

McQuarrie, F., \& Jackson, E. L. (1996). Connections between negotiation of leisure constraints and serious leisure: An exploratory study of adult amateur ice skaters. Loisir et societe/Society and Leisure, 19(2), 459-483.Available at: https://doi.org/10.1080/07053436.1996.10715528.

O'Connor, J. P., \& Brown, T. D. (2010). Riding with the sharks: serious leisure cyclist's perceptions of sharing the road with motorists. Journal of Science and Medicine in Sport, 13(1), 53-58.Available at: https://doi.org/10.1016/j.jsams.2008.11.003.

Ozdemir, A. S., Ayyıldız Durhan, T., \& Akgül, B. M. (2020). Serious leisure inventory and measurement (Short Form): Validity and reliability analysis. Asian Journal of Education and Training, 6(2), 207-212.

Patterson, I., Getz, D., \& Gubb, K. (2016). The social world and event travel career of the serious yoga devotee. Leisure Studies, 35(3), 296313.Available at: https://doi.org/10.1080/02614367.2014.962583.

Raisborough, J. (2007). Gender and serious leisure careers: A case study of women sea cadets. Journal of Leisure Research, 39(4), 686704.Available at: https://doi.org/10.1080/00222216.2007.11950128.

Robinson, R., Patterson, I., \& Axelsen, M. (2014). The "loneliness of the long-distance runner" no more: Marathons and social worlds. Journal of Leisure Research, 46(4), 375-394.Available at: https://doi.org/10.1080/002222 16.2014.11950333.

Romero, S., Iraurgi, I., Madariaga, A., \& Gould, J. (2020). Structural validity of the serious leisure inventory \& measure (SLIM) in different sets of athletes. Journal of Leisure Research, 1-16.Available at: https://doi.org/10.1080/002222 16.2019.1710307.

Ronkainen, N. J., Harrison, M., Shuman, A., \& Ryba, T. V. (2017). China, why not?: serious leisure and transmigrant runners' stories from Beijing. Leisure Studies, 36(3), 371-382.

Sherry, A., \& Bartram, M. A. (2001). Serious leisure careers among whitewater kayakers: A feminist perspective. World Leisure Journal, 43(2), 4-11.Available at: https://doi.org/10.1080/04419057.2001.9674225.

Shipway, R., \& Jones, I. (2007). Running away from home: Understanding visitor experiences and behaviour at sport tourism events. International Journal of Tourism Research, 9(5), 373-383.Available at: https://doi.org/10.1002/jtr.641.

Siegenthaler, K. L., \& Gonzalez, G. L. (1997). Youth sports as serious leisure: A critique. Journal of Sport and Social Issues, 21(3), 298314.Available at: https://doi.org/10.1177/019372397021003006.

Siegenthaler, K. L., \& O’Dell, I. (2003). Older golfers: Serious leisure and successful aging. World Leisure Journal, 45(1), 47-54. 
Stebbins, R. A. (2007). Serious leisure: A perspective for our time. New Brunswick, NJ: Transaction.

Stebbins, R. A. (2015). Leisure and the motive to volunteer: Theories of serious, casual, and project-based leisure. Basingstoke, UK: Palgrave Macmillan.

Stebbins, R. A. (1996). The barbershop singer: Inside the social world of a musical hobby. Toronto: University of Toronto Press.

Stebbins, R. A. (2009). Serious leisure and work. Sociology Compass, 3(5), 764-774.

Stebbins, R. A. (1992). Amateurs, professionals and serious leisure. Montréal, Canada: McGill-Queen's University Press.

Stebbins, R. A. (2014). Careers in serious leisure: From dabbler to devotee in search offulfillment. Basingstoke, UK: Palgrave Macmillan.

Stebbins, R. A. (1982). Serious leisure: A conceptual statement. Pacific Sociological Reviere, 25(2), 251-272.Available at: https://doi.org/10.2307/1388726.

Stebbins, R. A. (2001). New directions in the theory and research of serious leisure. Lewiston, NY: Edwin Mellen Press.

Stebbins, R. A. (2012). The idea of leisure. New Brunswick, NJ: Transaction.

Veal, A. J. (2017). The serious leisure perspective and the experience of leisure. Leisure Sciences, 39(3), 205-223. 